\title{
El tratamiento de la morfología en Primaria. Una propuesta desde la psicolingüística y la adquisición*
}

\author{
Morphology in Primary School. A Proposal from \\ Psycholinguistics and Language Acquisition
}

\section{Silvia Gumiel-Molina}

Universidad de Alcalá

silvia.gumiel@uah.es

ORCID ID: https://orcid.org/0000-0002-3918-0489

\section{Isabel Pérez-Jiménez}

Universidad de Alcalá

isabel.perezj@uah.es

ORCID ID: https://orcid.org/0000-0002-3040-3906

DOI: $10.17398 / 1988-8430.35 .1 .71$

Gumiel-Molina, S, y Pérez-Jiménez, I. (2022). El tratamiento de la morfología en Primaria. Una propuesta desde la psicolingüística y la adquisición. Tejuelo, 35(1), 71-106.

Doi: https://doi.org/10.17398/1988-8430.35.1.71

\footnotetext{
${ }^{*}$ Este trabajo se ha realizado en el marco del Proyecto Evidentiality, perspectivization and subjectivization at the interfaces of language - EPSILone (PID2019-104405GB-I00). Agradecemos a Carmen Fernández López, Norberto Moreno-Quibén, David Serrano Dolader y Adriana Soto-Corominas sus aportaciones bibliográficas y sus comentarios sobre este trabajo. A Josefa Martín García, le agradecemos, además, su atenta labor como responsable de este monográfico. Gracias también a Antonio García Gómez por su ayuda con el idioma y revisión de estilo. Debe también agradecerse el minucioso y cuidado trabajo de los editores de la revista Tejuelo.
} 
Resumen: El estudio explícito de la gramática en Educación Primaria es un asunto de debate permanente entre maestros y entre lingüistas. En este artículo se defiende la enseñanza explícita de la morfología en Primaria ya que contribuye a desarrollar la competencia comunicativa del alumnado: potencia la adquisición de vocabulario, aumenta la competencia lectora y mejora la expresión escrita. Para enseñar morfología con este objetivo comunicativo es necesario replantear los contenidos que se establecen en la ley educativa y el modo en que estos se presentan y se trabajan en los materiales diseñados para el aula. En este sentido, este trabajo ofrece una revisión crítica de esos dos aspectos y propone una organización de contenidos de morfología para la etapa de Primaria.

Keywords: morfología; Educación Primaria; competencia comunicativa; LOMCE.

\begin{abstract}
Explicit grammar instruction in primary schools encourages and sustains on-going debate among educators and linguists. This article argues that Morphology must be explicitly taught at primary level insofar as this type of instruction: increases vocabulary acquisition; improves students' reading comprehension skills; and enhances students' writing skills. Such an approach to the teaching of Morphology involves not only rethinking the contents that are established in the standing educational legislation but also adapting the way in which teaching materials are exploited in the classroom. Therefore, the present study offers a critical review of these aspects and proposes a possible way to organise the curricular content at primary level.
\end{abstract}

Palabras clave: morphology; Primary Education; communicative competence; LOMCE. 


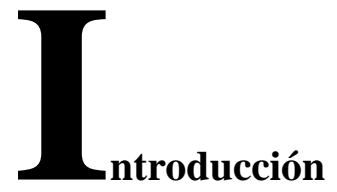

La necesidad de la reflexión gramatical en la Educación Primaria, los contenidos gramaticales que deben abordarse y las perspectivas metodológicas que han de adoptarse son asuntos sujetos a debate permanente tanto entre los lingüistas como entre los maestros de Lengua Castellana de esta etapa educativa. En el contexto de este debate, este artículo defiende que enseñar contenidos morfológicos en Primaria de forma explícita permite mejorar la capacidad reflexiva del alumnado y también sus capacidades comunicativas.

Para justificar esta posición, el artículo se organiza como sigue: en el primer apartado se expone cómo la LOMCE introduce la reflexión sobre el componente morfológico de forma progresiva a lo largo de la etapa de Primaria con el fin de mejorar las destrezas de comunicación. 
En el segundo apartado se analiza cómo se reflejan en los libros de texto los planteamientos plasmados en la ley, así como sus limitaciones e incoherencias. En el apartado 3, se defiende que el trabajo explícito de la conciencia morfológica promueve la adquisición del vocabulario y mejora las capacidades lectoescritoras, tal como se ha demostrado desde el campo de la adquisición de lenguas (L1 y L2) y del procesamiento del léxico. A partir de esta postura teórica, en el apartado cuatro, se sostiene que la reflexión explícita sobre el componente morfológico es necesaria y beneficiosa en Primaria, pero debe llevarse a cabo de manera pautada. La pauta vendrá marcada por lo que se sabe sobre el proceso de adquisición de la lengua materna y sobre la maduración de las capacidades de reflexión metalingüística del alumnado. En el apartado 5, y a la luz de las conclusiones anteriores, se presenta una propuesta de secuenciación de contenidos para la enseñanza de la morfología en la Educación Primaria.

\section{1.- La enseñanza de la morfología en la Educación Primaria. La LOMCE}

La LOMCE, tal como se indica en el Currículo básico de Primaria ${ }^{1}$, pretende que el alumnado desarrolle las siete competencias clave definidas por la UE, entre las cuales se encuentra la Competencia en comunicación lingüística.

Esta competencia se trabaja en Primaria principalmente a través del área troncal de conocimiento de Lengua Castellana y Literatura ${ }^{2}$ (en adelante LCL). El objetivo específico de esta área es "aportarle [al alumno] las herramientas y los conocimientos necesarios para desenvolverse satisfactoriamente en cualquier situación comunicativa de la vida familiar, social y profesional. Esos conocimientos son los que articulan los procesos de comprensión y expresión oral, por un lado, y de comprensión y expresión escrita, por otro" (Currículo, p. 26).

\footnotetext{
${ }^{1}$ Real Decreto 126/2014, de 28 de febrero; BOE, número 52, 1 de marzo de 2014; BOE-A-2014-2222; https://www.boe.es/buscar/pdf/2014/BOE-A-2014-2222consolidado.pdf

${ }_{2}^{2}$ En este artículo se deja de lado la enseñanza de la Literatura.
} 
Niños y niñas, por tanto, deben adquirir conocimientos y herramientas lingüísticos que les permitan poner en ejecución las cuatro destrezas para comunicarse adecuadamente en distintos tipos de situaciones sociales y contextos lingüísticos (incluyendo su formación como lectores cultos y competentes). Esos conocimientos y herramientas, además, deben permitir al alumnado continuar con aprovechamiento las etapas posteriores de su aprendizaje (Currículo, p. $3)$.

El foco de la enseñanza de la lengua, por tanto, apunta a la capacitación del alumnado para la resolución de tareas comunicativas mediante el desarrollo de las destrezas básicas y el conocimiento de los aspectos prescriptivos de la lengua.

Para desarrollar de forma parcelada los objetivos del área de LCL, los contenidos, criterios de evaluación y estándares de aprendizaje se dividen en cinco Bloques: 1. Comunicación oral: hablar y escuchar; 2. Comunicación escrita: leer; 3. Comunicación escrita: escribir; 4. Conocimiento de la lengua, y 5. Educación literaria.

La finalidad específica del bloque de 'Conocimiento de la lengua' es desarrollar la capacidad de reflexión del alumnado sobre el sistema linguístico como medio para alcanzar los objetivos comunicativos del área:

El Bloque 4, Conocimiento de la lengua, responde a la necesidad de reflexión sobre los mecanismos lingüísticos que regulan la comunicación, y se aleja de la pretensión de utilizar los conocimientos lingüísticos como un fin en sí mismos para devolverles su funcionalidad original: servir de base para el uso correcto de la lengua. El Conocimiento de la Lengua dentro del aula de Educación Primaria se plantea como el aprendizaje progresivo de las habilidades lingüísticas, así como la construcción de competencias en los usos discursivos del lenguaje a partir del conocimiento y la reflexión necesarios para apropiarse de las reglas gramaticales y ortográficas, imprescindibles, para hablar, leer y escribir correctamente en todas las esferas de la vida.

(LOMCE, Introducción al Bloque 4 'Conocimiento de la lengua’, p. 28). 
Estas afirmaciones plasman el enfoque instrumental y prescriptivo de la lengua que subyace a toda la ley educativa y que plantea en sí mismo problemas, como han señalado, entre otros, Bosque y Gallego $(2016,2018)$. No se profundizará en estos problemas aquí y se asumirá, en consonancia con la ley, que el objetivo fundamental del bloque de 'Conocimiento de la lengua' en Primaria ha de ser promover la reflexión sobre el sistema lingüístico para mejorar las capacidades comunicativas del alumnado: reflexión para la comunicación.

Sin embargo, la propia ley condiciona la consecución de este objetivo. Considérense los contenidos, criterios de evaluación y estándares de aprendizaje del Bloque 4 'Conocimiento de la lengua', recogidos en la Imagen 1 (se resaltan en amarillo los relacionados con la morfología). En la columna de estándares de aprendizaje evaluables, puede observarse que los verbos más repetidos son reconoce e identifica, lo que promueve que en el aula se haga meramente identificación, taxonomía y clasificación de unidades lingüísticas de forma mecánica e irreflexiva. Los estándares de aprendizaje, que guían la evaluación $y$, por ende, el modo de enfocar los contenidos, no favorecen el desarrollo de la reflexión linguiística, pese a lo señalado en el texto de la ley. Tampoco favorecen, pese a la definición del objetivo del área de LCL, la conexión de los contenidos con el desarrollo de la competencia comunicativa. 


\section{Imagen 1}

\section{Currículo básico de la Educación Primaria}

\begin{tabular}{|c|c|c|}
\hline Contenidos & Criterios de evaluación & Estándares de aprendizaje evaluables \\
\hline $\begin{array}{l}\text { La palabra. } \\
\text { Reconocimiento de las distintas clases de palabras y } \\
\text { explicación reflexiva de su uso en situaciones } \\
\text { concretas de comuninicación (nombre, verbo, adjetivo, } \\
\text { preposición, adverbio, conjunción, pronombres, } \\
\text { articulos, interjecciones). Características y uso de cada } \\
\text { clase de palabra. } \\
\text { Clases de nombres: comunes, propios, individuales, } \\
\text { colectivos, concretos y abstractos. } \\
\text { Conjugación de los verbos regulares e irregulares más } \\
\text { frecuentes. } \\
\text { La sílaba. Diptongos e hiatos. } \\
\text { Vocabulario: sinónimos y antónimos homónimos y } \\
\text { palabras polisémicas. Aumentativos y diminutivos. } \\
\text { Arcaismos, neologismos y extranjerismos. Frases } \\
\text { hechas. Formación de sustantivos, adjetivos y verbos. } \\
\text { Recursos derivativos: preffjos y sufijos en la formación } \\
\text { de nombres, adjetivos y verbos. Siglas y abreviaturas. } \\
\text { Uso eficaz del diccionario para ampliación de } \\
\text { vocabulario y como consulta ortográica y gramatical. } \\
\text { Ortografía: utilización de las reglas básicas de } \\
\text { ortografía. Reglas de acentuación. Signos de } \\
\text { puntuación. } \\
\text { Las relaciones gramaticales. } \\
\text { Reconocimiento y explicación reflexiva de las } \\
\text { relaciones que se establecen entre el sustantivo y el } \\
\text { resto de los componentes del grupo nominal. } \\
\text { Reconocimiento y observación reflexiva de los } \\
\text { constituyentes oracionales: la oración simple, sujeto y } \\
\text { predicado. } \\
\text { Reconocimiento y uso de algunos conectores textuales } \\
\text { (de orden, contraste y explicación) y de los principales } \\
\text { mecanismos de referencia interna, tanto gramaticales } \\
\text { (sustituciones pronominales) como léxicos (elipsis y } \\
\text { sustituciones mediante sinónimos e hiperónimos). } \\
\text { Las variedades de la lengua. } \\
\text { Conocimiento general de realidad plurilingūe de } \\
\text { España y su valoración como fuente de } \\
\text { enriquecimiento personal y como una muestra de la } \\
\text { riqueza de nuestro pattimonio histórico y cultural. }\end{array}$ & $\begin{array}{l}\text { 1. Aplicar los conocimientos básicos sobre la estructura de la } \\
\text { lengua, la gramática (categorías gramaticales), el vocabulario } \\
\text { (formación y significado de las palabras y campos } \\
\text { semánticos), así como las reglas de ortografía para favorecer } \\
\text { una comunicación más eficaz. } \\
\text { 2. Desarrollar las destrezas y competencias lingüísticas a } \\
\text { través del uso de la lengua. } \\
\text { 3. Sistematizar la adquisición de vocabulario a través de los } \\
\text { textos. } \\
\text { 4. Desarrollar estrategias para mejorar la comprensión oral y } \\
\text { escrita a través del conocimiento de la lengua. } \\
\text { 5. Utilizar programas educativos digitales para realizar tareas } \\
\text { y avanzar en el aprendizaje. } \\
\text { 6. Conocer la variedad linguística de España y del español } \\
\text { como fuente de enriquecimiento cultural. Mostrar respeto } \\
\text { tanto hacia las lenguas y dialectos que se hablan en España, } \\
\text { como hacia el español de América. }\end{array}$ & 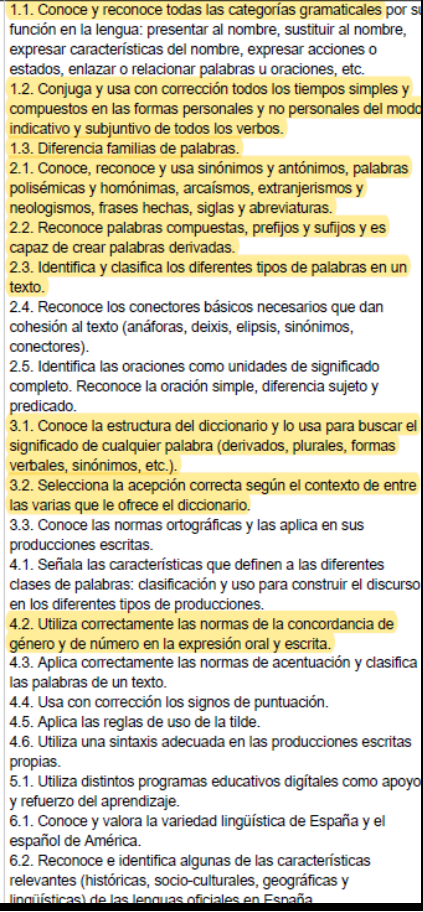 \\
\hline
\end{tabular}

Fuente: Real Decreto 126/2014, de 28 de febrero, por el que se establece el currículo básico de la Educación Primaria, p. 32

Junto con la definición de los estándares de aprendizaje, hay otro aspecto de la ley que influye en que los contenidos lingüísticos se planteen en la práctica desligados de la reflexión para la mejora de la comunicación: el enorme espectro conceptual que ha de cubrirse en esta etapa, que favorece su enseñanza puntual y estanca. Como se desprende de la Imagen 1, los contenidos relacionados con la morfología que se abarcan en Primaria se agrupan del siguiente modo: a) las categorías gramaticales (se incluye identificar todas las clases de palabras y sus subclases, así como conocer las propiedades morfosintácticas de cada una de ellas); b) los procesos de formación de palabras; y c) las relaciones paradigmáticas entre palabras; además del uso del diccionario. Respecto a su organización, la LOMCE propone que estos contenidos se organicen "de manera creciente en las distintas etapas y 
[supongan] una progresión respecto a los saberes y habilidades adquiridos desde el inicio de la vida escolar". No obstante, no se proporciona ninguna pauta específica respecto a esa organización progresiva.

En el siguiente apartado se analizará cómo se reflejan en los libros de texto estas incongruencias de la ley: desconexión entre la supuesta finalidad comunicativa del Bloque de 'Conocimiento de la lengua' y los estándares de aprendizaje que se definen; ausencia de una perspectiva reflexiva en la definición de esos estándares y falta de pautas o criterios claros para establecer la progresión de los contenidos señalada como necesaria.

\section{2.- La enseñanza de la morfología en los libros de texto. Limitaciones}

En este apartado se mostrará cómo la definición de los estándares de aprendizaje de la LOMCE, el volumen de contenidos que impone y la falta de concreción en su progresión pueden afectar al tratamiento de la morfología en los materiales que el alumnado utiliza en Primaria ${ }^{3}$. Para poder ofrecer ejemplos concretos, se han revisado los libros del alumno de Lengua castellana y Literatura de la editorial Santillana, Proyecto Saber hacer, desde tercero a sexto de Primaria. La elección de este material radica en su amplia difusión a nivel nacional y en su valoración positiva por la comunidad educativa. Los ejemplos seleccionados deben entenderse como una muestra del acercamiento a los contenidos y del tipo de actividades que son comunes en los materiales de Primaria más comercializados y difundidos en España.

${ }^{3}$ Para una reflexión sobre el estudio de la morfología y el modo en que se aborda en los libros de texto en Enseñanza Secundaria, consúltese Saragossà (2015). 


\section{1.- La falta de progresión}

Obsérvese la distribución de los contenidos relativos a la formación de palabras desde tercero a sexto de Primaria en los libros de texto mencionados (sección de Vocabulario).

\section{Tabla 1}

Contenidos relativos a la formación de palabras. Santillana - Saber hacer

\begin{tabular}{|c|c|c|c|c|}
\hline & Tercero & Cuarto & Quinto & Sexto \\
\hline T.1 & $\begin{array}{l}\text { Palabras } \\
\text { sinónimas }\end{array}$ & $\begin{array}{l}\text { Sinónimos y } \\
\text { antónimos }\end{array}$ & $\begin{array}{l}\text { Palabras } \\
\text { sinónimas y } \\
\text { antónimas }\end{array}$ & Prefijos y sufijos \\
\hline T.2 & $\begin{array}{l}\text { Palabras } \\
\text { antónimas }\end{array}$ & Palabras polisémicas & $\begin{array}{c}\text { Palabras } \\
\text { monosémicas y } \\
\text { polisémicas }\end{array}$ & $\begin{array}{l}\text { Prefijos y sufijos } \\
\text { intensivos }\end{array}$ \\
\hline T.3 & $\begin{array}{c}\text { Palabras } \\
\text { polisémicas }\end{array}$ & Palabras homófonas & $\begin{array}{l}\text { Palabras } \\
\text { homónimas }\end{array}$ & $\begin{array}{l}\text { Prefijos de } \\
\text { negación y } \\
\text { oposición }\end{array}$ \\
\hline T.4 & Frases hechas & $\begin{array}{c}\text { Palabras primitivas } \\
\text { y derivadas }\end{array}$ & $\begin{array}{l}\text { Palabras } \\
\text { parónimas }\end{array}$ & $\begin{array}{l}\text { Prefijos de } \\
\text { situación }\end{array}$ \\
\hline T.5 & Refranes & $\begin{array}{l}\text { Palabras simples y } \\
\text { compuestas }\end{array}$ & Campo semántico & $\begin{array}{l}\text { Formación de } \\
\text { sustantivos }\end{array}$ \\
\hline T.6 & $\begin{array}{l}\text { Palabras } \\
\text { derivadas }\end{array}$ & $\begin{array}{c}\text { Familias de } \\
\text { palabras }\end{array}$ & Campo léxico & $\begin{array}{l}\text { Formación de } \\
\text { adjetivos }\end{array}$ \\
\hline T.7 & $\begin{array}{l}\text { Palabras } \\
\text { compuestas }\end{array}$ & Prefijos y sufijos & $\begin{array}{c}\text { Palabras } \\
\text { compuestas y } \\
\text { simples }\end{array}$ & $\begin{array}{l}\text { Formación de } \\
\text { verbos }\end{array}$ \\
\hline T.8 & Diminutivos & $\begin{array}{l}\text { Sufijos diminutivos } \\
\text { y aumentativos }\end{array}$ & $\begin{array}{c}\text { Palabras } \\
\text { derivadas y } \\
\text { primitivas }\end{array}$ & $\begin{array}{c}\text { Palabras } \\
\text { onomatopéyicas }\end{array}$ \\
\hline T.9 & Aumentativos & $\begin{array}{c}\text { Prefijos de negación } \\
\text { y de lugar }\end{array}$ & $\begin{array}{l}\text { Familias de } \\
\text { palabras }\end{array}$ & $\begin{array}{l}\text { Las siglas y las } \\
\text { abreviaturas }\end{array}$ \\
\hline T.10 & $\begin{array}{c}\text { Familias de } \\
\text { palabras }\end{array}$ & Otros prefijos & Los prefijos & $\begin{array}{l}\text { Palabras tabú y } \\
\text { eufemismos }\end{array}$ \\
\hline T.11 & Gentilicios & Campo léxico & Los sufijos & $\begin{array}{c}\text { Las palabras } \\
\text { coloquiales }\end{array}$ \\
\hline T.12 & $\begin{array}{l}\text { Palabras } \\
\text { colectivas }\end{array}$ & Campo semántico & $\begin{array}{c}\text { Aumentativos y } \\
\text { diminutivos }\end{array}$ & $\begin{array}{c}\text { Arcaísmos, } \\
\text { extranjerismos y } \\
\text { neologismos }\end{array}$ \\
\hline T.13 & $\begin{array}{c}\text { Palabras } \\
\text { onomatopéyicas }\end{array}$ & Gentilicios & Los gentilicios & \\
\hline T.14 & $\begin{array}{l}\text { Palabras } \\
\text { parónimas }\end{array}$ & Frases hechas & Las frases hechas & \\
\hline T.15 & $\begin{array}{l}\text { Campo } \\
\text { semántico }\end{array}$ & $\begin{array}{c}\text { Siglas y } \\
\text { abreviaturas }\end{array}$ & Los refranes & \\
\hline
\end{tabular}

Fuente: elaboración propia 
Los contenidos relativos a la formación de palabras se agrupan en cuatro grandes temas, según orden de aparición: a) los procesos de derivación (que se estudian en tercero, cuarto y quinto y se señalan en azul), b) los procesos de composición (que se estudian en tercero, cuarto y quinto y aparecen en color crema), c) el estudio de los prefijos y los sufijos (color salmón) y, dentro de este, d) el análisis de prefijos y sufijos concretos (en color gris), con especial atención a aumentativos y diminutivos (en tercero, cuarto y quinto, en color verde). Las familias de palabras se tratan de forma independiente, así como otros mecanismos de formación de palabras (siglas).

Si se atiende a la mera formulación de los contenidos, no parece existir orden o progresión. Podría ocurrir que la complejidad teórica de los contenidos se incrementara con el avance de los cursos, pero la revisión realizada de los libros del alumno no parece apuntar en ese sentido. A modo de ejemplo se reproducen los contenidos de cuarto y sexto relativos a los prefijos de negación y de lugar ${ }^{4}$.

+Cuarto curso, Tema 9. Vocabulario. Prefijos de negación y de lugar.

"Los prefijos de negación se añaden a algunas palabras para formar otras de significado contrario. Por ejemplo, el prefijo des- se añade a la palabra animado para formar su antónimo desanimado. Algunos prefijos de negación son $a$-, des- o -in.

Los prefijos de lugar sirven para indicar situación. Por ejemplo, el prefijo subsignifica "por debajo" y sirve para formar palabras como subcampeón o submarino. Algunos prefijos de lugar son ante-, extra-, intra-, sub- o sobre-."

+Sexto curso, Tema 3. Vocabulario. Prefijos de negación y oposición.

"Los prefijos de negación se ponen delante de determinadas palabras para formar sus antónimos. Son prefijos de este tipo $a$-, in- y des-. Por ejemplo: atemporal, incomprensible, desagradable.

Otros prefijos se unen a ciertas palabras para dar la idea de oposición. Son los prefijos de oposición, como contra- y anti-. Por ejemplo: contracorriente y antihigiénico."

${ }^{4}$ El texto que es reproducción literal de los contenidos de los libros de texto revisados aparece en cuerpo menor. 
+Sexto curso. Tema 4. Vocabulario. Prefijos de situación.

"Los prefijos de situación indican lugar o situación. Por ejemplo, el prefijo sub- significa "debajo de": sub + campeón $\rightarrow$ subcampeón.

Otros prefijos de situación son los siguientes:

- sobre- (encima de). Por ejemplo: sobrevolar.

- ante-(delante de). Por ejemplo: antesala.

- extra- (fuera de). Por ejemplo: extraescolar."

Como se observa, los contenidos teóricos de cuarto y sexto reproducen básicamente la misma información y ofrecen ejemplos que son de complejidad similar, quizá con alguna excepción, como podría ser la inclusión de extraescolar, que denota un lugar abstracto o no locativo, lo que podría justificar el cambio terminológico prefijo de lugar > prefijo de situación (agradecemos a Carmen Fernández López esta puntualización $)^{5}$. Así, incluso entendiendo que la progresión en la dificultad radicara no en la formulación teórica de los contenidos sino en la contextualización y uso de los ejemplos planteados, no parece que esta pueda ser percibida como tal por el alumnado. Cabe señalar, además, que los prefijos locativos son los menos productivos en español y los que mayor lexicalización muestran. Los prefijos más productivos en español son los gradativos (súper-, requete-, re-), que no se tratan hasta sexto, aunque se han tratado los diminutivos y aumentativos desde tercero (agradecemos a Josefa Martín García esta observación, que cobrará relevancia más adelante).

${ }_{5}$ Tal y como nos hace notar un revisor anónimo, los libros de texto han de cuidar también los términos ejemplificadores con los que ilustran los contenidos. Así, por ejemplo, el libro de cuarto curso señala que "el prefijo des- se añade a la palabra animado para formar su antónimo desanimado". Nótese, no obstante, que esta no es la derivación adecuada, sino que a partir de 'animar' se forma 'desanimar' y posteriormente 'desanimado'. Algo similar sucede, como se señala en la siguiente nota, con la palabra callejón. La segmentación morfológica errónea parece, de hecho, un problema recurrente en los libros de texto. Agradecemos a este revisor este importante comentario. 


\section{2.- Contenidos presentados en compartimentos estancos}

Junto con lo señalado en el apartado anterior, la falta de relación explícita entre contenidos dificulta su aprendizaje. Considérense los contenidos 'palabras primitivas y derivadas', 'palabras simples y compuestas' y 'prefijos y sufijos', que se tratan en los temas 4, 5 y 7 de cuarto curso de los materiales revisados.

+ Cuarto curso. Tema 4. Vocabulario. Palabras primitivas y derivadas.

"Algunas palabras se han formado a partir de otras. Por ejemplo, a partir de la palabra calle se ha formado la palabra callejón $n^{6}$.

Las palabras que se han formado a partir de otras son palabras derivadas. Las palabras que no se han formado a partir de otras son palabras primitivas."

+Cuarto curso. Tema 5. Vocabulario. Palabras simples y compuestas.

"Las palabras compuestas son las que se han formado uniendo dos o más palabras. Por ejemplo, salvavidas se ha formado a partir de las palabras salva y vidas. A veces, al unir dos palabras para formar una compuesta, las palabras originales pueden sufrir algún cambio. Por ejemplo, agridulce (de agrio + dulce).

Las palabras simples, en cambio, no se componen de otras palabras. Por ejemplo, ejercicio."

+ Cuarto curso. Tema 7. Vocabulario. Prefijos y sufijos.

"Los prefijos y los sufijos son partículas que se añaden a algunas palabras para formar otras nuevas.

- Los prefijos se añaden delante de las palabras. Por ejemplo, a partir de la palabra vivir se han formado convivir, revivir, etc.

- Los sufijos, en cambio, se añaden detrás de las palabras. Por ejemplo, a partir de casa se han formado palabras como casero, casona o caserío."

${ }^{6}$ Nótese que la forma callejón no se forma a partir de calle, sino de calleja. Estos errores dificultan la reflexión e inducen la formación de hipótesis erróneas: en este caso, la hipótesis de que el sufijo aumentativo es -ejón. Agradecemos a Josefa Martín García esta observación. 
Si bien los contenidos anteriores están ligados desde el punto de vista teórico y conceptual, no se establece conexión explícita entre ellos en el libro del alumno; por ejemplo, no se señala de forma explícita la relación que existe entre la adición de prefijos y sufijos y la formación de palabras derivadas. Esta desconexión dificulta la reflexión y el establecimiento de vínculos significativos entre contenidos, que habrán de ser aportados por el docente o inferidos por el estudiante. Nótese, además, que tampoco se exploran las diferencias y semejanzas entre los procesos de derivación y composición, ni la relación entre flexión y derivación en conexión con la noción de palabra. Sin embargo, el establecimiento explícito de este tipo de relaciones es crucial para fomentar la reflexión y para desarrollar la conciencia morfológica.

\section{3.- Falta de reflexión y desconexión de la comunicación}

El último de los problemas detectados en el Currículo que se refleja en los libros de texto del alumno es que los ejercicios propuestos son escasamente reflexivos. Las actividades que acompañan a las explicaciones teóricas no se basan, generalmente, en la comparación o contraste de unidades lingüísticas o en la búsqueda de generalizaciones, sino en la mera aplicación de las definiciones dadas en tareas de segmentación. No hay lugar para la creación del alumnado de sus propias muestras de lengua contextualizadas sobre las que reflexionar y tampoco se orientan las actividades al desarrollo de la competencia comunicativa (a excepción, quizá, de los ejercicios de búsqueda en el diccionario). Se ejemplificará este punto con algunos ejercicios del tema cuatro de sexto curso de los materiales revisados, dedicado a los prefijos de situación. 
+Sexto curso. Tema 4. Vocabulario. Prefijos de situación

1. Forma palabras con estos prefijos:

\begin{tabular}{|c|c|c|l|}
\hline Sub- & suelo & marino & título \\
\hline Ante- & poner & penúltimo & proyecto \\
\hline
\end{tabular}

5. Analiza las palabras según el ejemplo.

- Subestimar $\rightarrow$ sub + estimar

- $\quad$ Sobrefalda $\rightarrow$

- $\quad$ Extrarradio $\rightarrow$

- Anteojos $\rightarrow$

6. Copia estas palabras y rodea los prefijos

- Subdelegado

- Extralimitarse
- Sobreedificar

- Antepasado

Este tipo de ejercicios, frecuentes en diferentes materiales, no mejora la competencia comunicativa del alumnado en ningún sentido ni tampoco permite saber si este ha entendido o no cómo tienen lugar los procesos de formación de palabras; un alumno puede formar submarino o antepenúltimo y rodear el prefijo sub- en subdelegado aun sin saber qué significan estas palabras. Del mismo modo, un alumno podrá identificar el prefijo extra- en extrarradio sin entender el significado de esta palabra (probablemente, no conocerá el significado de radio o lo aplicará a la circunferencia, pero no a una población). Tampoco contribuyen estas actividades al aumento del vocabulario de los estudiantes que, probablemente, no van a utilizar palabras como sobrefalda.

Si bien no es el objetivo de este artículo proponer un conjunto de actividades reflexivas, no queremos dejar de señalar que los ejercicios en los que el estudiante tenga que inventar nuevas palabras y explicar su significado (subasfalto, sobrecielo, etc.) o actividades de búsqueda de palabras ("vamos a decir palabras que empiecen con 'sub-' e intentaremos descubrir su significado") son mejores en tanto que propician la reflexión sobre la lengua, contribuyen a aumentar el 
vocabulario de los estudiantes y, además, resultan, por lo general, más interesantes y motivadores ${ }^{7}$.

Tras esta breve revisión, tomada como botón de muestra del modo en que se enfocan comúnmente los contenidos y actividades en los libros del alumno de Lengua Castellana y Literatura de Primaria, parece poder concluirse que la enseñanza de la morfología en esta etapa se plantea de manera no progresiva, no reflexiva y desconectada de la comunicación.

Sin embargo, como se muestra en el siguiente apartado, la reflexión explícita sobre la morfología en Educación Primaria (esto es, el desarrollo de la conciencia morfológica) resulta fundamental para la mejora de la competencia comunicativa en lo que respecta al aumento del vocabulario del alumnado y al progreso de la lectoescritura.

\section{3.- El desarrollo de la conciencia morfológica contribuye a la mejora de la competencia comunicativa}

Considérense de nuevo los contenidos sobre procesos de formación de palabras que han de trabajarse en Educación Primaria según el Currículo:

Vocabulario: aumentativos y diminutivos, neologismos y extranjerismos. Formación de sustantivos, adjetivos y verbos. Recursos derivativos: prefijos y sufijos en la formación de nombres, adjetivos y verbos. Siglas y abreviaturas (contenidos relacionados son la conjugación verbal y el uso del diccionario).

Estos contenidos se ligan en la ley con las siguientes finalidades comunicativas:

\footnotetext{
${ }^{7}$ De hecho, tal y como nos señala un revisor anónimo, la mejor forma de que los niños aprendan palabras nuevas no es el análisis de palabras ya formadas sino el vocabulario potencial; esto es, permitir que los niños jueguen con el lenguaje creando palabras posibles. Como nos señala este revisor, se puede preguntar el nombre de la persona que trabaja en una copistería (copistero) o de alguien que destruye discos duros (discoduricida). Agradecemos a este revisor su comentario.
} 
-favorecer una comunicación más eficaz,

-desarrollar las destrezas lingüísticas a través del uso de la lengua, -sistematizar la adquisición de vocabulario a través de los textos, -desarrollar estrategias para mejorar la comprensión oral y escrita.

La pregunta pertinente ahora es si la reflexión explícita sobre la palabra y su estructura interna - el desarrollo de la conciencia morfológica - permite alcanzar estos objetivos.

La respuesta dada desde el campo de la adquisición y el procesamiento de la lengua, como se mostrará a continuación, es sí. El desarrollo de la conciencia morfológica contribuye de manera directa a mejorar, al menos, tres aspectos básicos de la comunicación: la adquisición y procesamiento del vocabulario, la competencia lectora y la competencia escrita.

\section{1.- Morfología y adquisición/procesamiento de vocabulario}

Bloom (2000, 2001) señala (para el inglés) que, entre el primer año de vida y el final de la adolescencia, hacia los 17 años, una persona ha aprendido unas 60.000 palabras. Por otro lado, Clark (2015) afirma que a los seis años los niños manejan aproximadamente un vocabulario de unas 14.000 palabras. Así pues, en la etapa de la formación obligatoria (Primaria y Secundaria) el niño/adolescente aprende unas 46.000 palabras, lo que supone una media de más de 11 palabras diarias. Parece, no obstante, que este aprendizaje no es lineal, sino que aumenta progresivamente con la edad dentro de ese periodo. Según Bloom (2000, 2001), varios son los factores que influyen en esa progresión:

-La exposición del niño a la lengua. Las palabras se aprenden estableciendo redes entre ellas, definidas por distintos parámetros, que no se analizarán aquí; baste con mencionar redes basadas en la forma, en el significado o en los contextos de aparición de las palabras. Por tanto, cuanto mayor sea la exposición del niño a entornos linguísticos variados, más palabras podrá conocer, más redes podrá crear y, por 
tanto, más palabras nuevas podrá conectar a estas redes. En este sentido, Bloom $(2000,2001)$ señala el momento de acceso a la lectura como un punto de inflexión en la adquisición de vocabulario.

-Las capacidades perceptivas del niño. Cuanto mayor es el niño, mayor es su capacidad de procesar nuevas experiencias, identificando 'acciones', 'objetos', etc., lo que le permite aprender nuevas palabras asociadas a ellas (e insertarlas en redes).

-El aprendizaje de información relevante de las palabras. El aprendizaje de nuevos términos permite al niño establecer generalizaciones relativas a la forma-significado. Esta información facilita la adquisición más rápida de nuevas palabras; por ejemplo, cuando un niño aprende una nueva palabra acabada en -ción su competencia lingüística le permite identificar esta como un sustantivo femenino, de acción, con estructura eventiva y que designa entidades abstractas.

Como han sostenido distintos autores, el aprendizaje del léxico prosigue a lo largo de la vida. Sin embargo, se produce una disminución considerable en el número de palabras nuevas que se adquieren en la edad adulta, hecho ligado posiblemente a un descenso a la exposición a vocabulario nuevo (Bloom, 2000, 2001). Por todo ello, parece que el rango de edad que comprende la Educación Primaria (6 - 12 años) es óptimo para potenciar el aprendizaje de nuevo vocabulario. El aprendizaje de vocabulario es clave, a su vez, para el desarrollo de la producción y comprensión linguiística oral y escrita.

Debe, por tanto, considerarse ahora qué papel desempeña la información morfológica de las palabras en la adquisición del vocabulario, y específicamente, en el almacenamiento de nuevas formas en el lexicón, entendiendo este componente como el sistema de conocimiento del vocabulario al que el hablante accede en la producción y comprensión de la lengua.

Existen distintas teorías al respecto. La que nos interesa presentar aquí — defendida por Forster (1976), Morton (1979), Fromkin 
(1985) o Caramazza et al. (1988), i.a. - propone que el vocabulario se almacena en distintos subcomponentes léxicos interconectados. El lexicón, por tanto, se organiza en subcomponentes que contienen (al menos) información fonológica, ortográfica, semántica y sintáctica de las piezas de vocabulario. Los pacientes con trastornos del lenguaje proporcionan evidencia de la organización modular de estos componentes, como indican Raiter y Jaichenco (2002); así, ciertos tipos de afasia perceptiva implican problemas para identificar oralmente una palabra, pero no para leerla, mientras que determinadas alteraciones en la producción pueden afectar a la escritura, pero no a la producción oral. Esto parece mostrar que nuestro lexicón almacena separadamente, al menos, la información fonológica y la información ortográfica del vocabulario (posiblemente además de forma independiente en la producción y la comprensión). Otras afectaciones, como la anomia verbal/nominal o el agramatismo, indican que la categoría gramatical también se almacena de forma independiente de otras propiedades de la palabra.

¿Qué ocurre con la estructura morfológica de las palabras complejas o polimorfemáticas? ¿Es relevante la estructuración morfológica de la palabra para la adquisición de nuevo vocabulario y su almacenamiento en el lexicón? Como señalan Raiter y Jaichenco (2002), existen dos respuestas para esa pregunta. Por una parte, según la hipótesis de la lista completa, (Butterworth, 1983) las palabras polimorfemáticas se almacenan en nuestro lexicón en su forma completa. Por otra, la hipótesis de la descomposición morfológica (Taft y Forster, 1975; Sánchez Gutiérrez, 2012 para el español) propone que en la adquisición de palabras polimorfemáticas se producen procesos de descomposición que aíslan los morfemas de la forma completa y los almacenan separadamente, y, posteriormente, procesos de reintegración de esos morfemas que dan lugar a una representación (sintáctica y semántica) de la palabra completa. La existencia de pacientes con lesiones cerebrales que muestran afectación selectiva de bases o afijos parece apoyar que hay un almacenamiento separado de estos componentes morfológicos. 
La hipótesis de la descomposición morfológica presenta un lexicón económico y con capacidad generativa para la creación de significados, y parece ser una propuesta adecuada para el funcionamiento de la morfología flexiva regular. Según esta hipótesis, se almacenarían en el léxico la raíz y el paradigma de afijos flexivos. Esta propuesta, no obstante, debe también dar cuenta de cómo se almacenan las formas verbales o nominales con flexión irregular (quepo o el plural de crisis, por ejemplo), o las formas supletivas de algunos verbos (como fue para ser). Con relación a la morfología derivativa, debe explicar la no composicionalidad del significado de las formas completas como, por ejemplo, amable o pasamontañas.

Caramazza et al. (1988) concilian las dos hipótesis presentadas y defienden que todas las formas del vocabulario de una lengua, incluidas las palabras polimorfemáticas, tienen una representación completa en el léxico. Las palabras polimorfemáticas tienen, además, un almacenamiento descompuesto. En el acceso al léxico polimorfemático por parte del hablante, o en su procesamiento, ambas rutas son posibles, de modo que se accede a las palabras más frecuentes mediante la primera ruta (representación completa), mientras que la segunda (a través de los morfemas) se activa para las palabras poco frecuentes o nuevas, que el hablante no tiene almacenadas en el lexicón.

Por último, para comprender la importancia de la estructura morfológica en la adquisición y procesamiento del vocabulario, ha de recordarse que las palabras se almacenan en el lexicón estableciendo redes. Uno de los parámetros de establecimiento de redes es el significado. Forster (1976) propone que cuando se localiza la entrada de una palabra en el léxico se produce un proceso de facilitación (priming semántico) tal que "es posible acceder directamente [de forma interna al lexicón] a la entrada de otra relacionada semánticamente" (Raiter y Jaichenco, 2002). Así, el almacenamiento descompuesto de las palabras polimorfemáticas permitiría establecer este tipo de redes de significado a partir, no solo de formas completas, sino de raíces y sufijos. Como el lector podrá anticipar, este tipo de procesos son cruciales en el desarrollo, por ejemplo, de la competencia lectora. 
En conclusión, los resultados de investigación reseñados en este apartado muestran que la información morfológica es relevante en la adquisición y procesamiento del léxico. Si esto es así, puede esperarse que la reflexión sobre la estructura morfológica facilite el que los niños puedan reconocer, almacenar y recuperar nuevas palabras no escuchadas anteriormente. El consiguiente incremento del vocabulario permitirá mejorar su competencia comunicativa en las cuatro destrezas básicas (Martín Vegas, 2018; Hernández Muñoz, 2015 para una conclusión similar en adultos).

Esta idea existe desde hace tiempo en el campo de la enseñanza del español como lengua extranjera. Morín (2003), Sánchez Gutiérrez (2012), Serrano Dolader (2019), y Rodríguez Montes (2020), entre otros, han defendido la idea de que el desarrollo de la conciencia morfológica mediante la reflexión explícita sobre la estructura interna de las palabras — el "entrenamiento morfológico" — permite mejorar:

-el acceso a los términos almacenados en el lexicón,

-el establecimiento de redes formales y semánticas entre esos términos,

-las habilidades de inferencia léxica,

-la productividad léxica y, por último,

-la competencia lectoescritora.

A este último aspecto se dedicarán las siguientes secciones.

\section{2.- Morfología y desarrollo de la comprensión lectora}

Los distintos trabajos de Carlisle (Carlisle, 1988, 1995; Carlisle y Stone, 2003) y de Nagy (véase, entre otros, Nagy et al., 1993) mostraron que la capacidad de los niños de interpretar vocablos nuevos en tareas de lectura en Educación Primaria se ve incrementada si son capaces de utilizar su conocimiento sobre la estructura morfológica de las palabras. En los estadios iniciales de aprendizaje de la lectura, la mayoría de las palabras que los niños encuentran está ya en su léxico oral, por lo que la descodificación fonológica da acceso al niño a la pronunciación y al significado de la palabra. Sin embargo, conforme 
avanza la Educación Primaria, los niños se enfrentan a la lectura de palabras ausentes de su léxico oral que, sin embargo, se relacionan con aquellas mediante procesos morfológicos, por lo que requieren de estrategias de comprensión que aprovechen estas relaciones.

En este sentido, con relación al español, Rodrigo et al. (2004) y Jaichenco y Wilson (2013), entre otros, muestran cómo el reconocimiento de morfemas contribuye a un mejor procesamiento de la lectura tanto en lectores sin dificultades como en pacientes disléxicos, puesto que funciona como "un potente predictor de la comprensión" (Gómez Vera et al., 2014).

\section{3.- Morfología y desarrollo de la expresión escrita}

También parece existir relación entre el dominio del vocabulario por parte del niño y su expresión escrita, como afirman Gómez Vera et al. (2014). Una razón de esta relación, como señala McCutchen (2011), parece ser que el conocimiento y manejo del vocabulario por el niño permite disminuir la carga de la memoria de trabajo durante la producción del texto, que puede, así, dirigirse a otras microhabilidades de la escritura.

Por otra parte, el aumento del vocabulario influye en la calidad del texto, en cuanto a la sofisticación y la densidad léxica que muestra (Ávila, 1991; McNamara et al., 2010). Se entiende por sofisticación léxica el uso de un vocabulario poco frecuente, de palabras más largas y con mayor número de sílabas. La densidad léxica hace referencia al número de unidades con significado léxico presentes en un texto (entendiendo estas unidades como opuestas a las palabras funcionales, que tienen significado gramatical).

Por último, si el estudio de la morfología se basa en la observación y manipulación de unidades, resolución de problemas, generación y confirmación de hipótesis, etc., se estará contribuyendo, no solo al desarrollo de la competencia comunicativa (incremento del 
caudal léxico, mejora en las destrezas) sino, además, al desarrollo de la competencia estratégica.

\section{4.- Reflexión sí, pero ¿cómo? De nuevo una mirada desde la lingüística experimental}

En apartados anteriores se ha intentado justificar que el desarrollo de la conciencia morfológica en Primaria, definida como la capacidad del niño para reflexionar sobre la estructura de la palabra y para manipularla de forma consciente, tiene beneficios para la adquisición y procesamiento del vocabulario, y en consecuencia para la mejora de la lectura y de la expresión escrita. Hay que preguntarse, no obstante, si la conciencia morfológica está lo suficientemente desarrollada en los niños de Primaria como para poder plantear ejercicios explícitos de reflexión morfológica en el aula. En este apartado se expondrá cuál ha sido la respuesta a esta pregunta desde el campo de la adquisición y el procesamiento de la lengua.

Tyler y Nagy (1989) mostraron experimentalmente con niños anglófonos (inglés L1) de cuarto (8-9 años), sexto (10-11 años) y octavo (12-13 años) grado que la conciencia morfológica comprende un conjunto de conocimientos que se desarrollan secuencialmente.

El primer conocimiento al que los niños pueden acceder es el de identificar la presencia de unidades menores que la palabra que portan significado (morfemas) (usando un ejemplo del español, los niños pueden acceder al conocimiento de que -dor es una unidad con significado en jugador, corredor, nadador); es lo que se denomina conocimiento receptivo.

En segundo lugar, los niños son capaces de acceder a un conocimiento relacional, esto es, pueden reconocer que dos palabras comparten una misma base (jugar, jugador).

Posteriormente, se accede al conocimiento sintáctico, a través del cual los niños son capaces de darse cuenta de que ciertos sufijos 
poseen determinada categoría gramatical, de tal modo que, por ejemplo, observan que las palabras que acaban en -miento son nombres y las que acaban en -ble son adjetivos.

El último conocimiento que es accesible al niño es el de las restricciones de selección entre sufijos y bases (conocimiento distribucional).

El estudio de estos autores permitió extraer las siguientes conclusiones: a) Los niños desarrollan el conocimiento relacional antes del cuarto grado (9 años); b) Su conocimiento, al principio limitado, de las propiedades sintácticas de los sufijos aumenta progresivamente con la edad; y c) Su conocimiento de las limitaciones de distribución de los sufijos y las bases también aumenta progresivamente, y no se adquiere totalmente hasta el octavo grado (12-13).

Roy y Labelle (2007), entre otros autores, reproducen resultados similares sobre la conciencia morfológica de niños francófonos. Estas autoras observaron que, en primer y segundo grado de Primaria (6-8 años), los niños tienen suficientemente desarrollada la conciencia morfológica receptiva y relacional como para utilizarla explícitamente en la enseñanza del vocabulario. El resto de los conocimientos morfológicos no son igualmente accesibles a los niños de esa edad y hay diferencias individuales.

En lo que respecta al español, Salvo de Vargas e Isuani (2017) llegan a idénticas conclusiones. Mediante un estudio experimental para medir la conciencia morfoléxica en sufijación, concluyen que, en séptimo grado de escuela primaria en Argentina (12-13 años), el acceso a la competencia receptiva y relacional está ya plenamente desarrollado. Por el contrario, el acceso a los conocimientos de tipo sintáctico y distribucional está menos desarrollado (siendo el conocimiento distribucional al que menor acceso tienen los niños).

Así, a pesar de las diferencias morfológicas interlingüísticas, se advierte un desarrollo similar de la conciencia morfológica en los hablantes de inglés, francés y español y se puede concluir que la 
capacidad para la reflexión morfológica está activa en la edad de escolarización obligatoria, al menos en lo que respecta al conocimiento receptivo y relacional.

Si esto es así, esta capacidad habrá de optimizarse utilizando materiales de reflexión adecuados. Nuevamente, los estudios sobre cómo se adquiere la morfología y cómo se desarrolla la conciencia morfológica ofrecen la base para organizar y pautar la reflexión gramatical en este ámbito.

En lo que respecta a la adquisición de la morfología, Nagy et al. (1993) señalan, en relación con el inglés, que los niños dominan los sufijos flexivos y la composición antes que la sufijación derivativa (según mostraron también en sus trabajos seminales Berko, 1958 y Kernan y Blount, 1966). Por lo tanto, en la práctica escolar, deben distinguirse y secuenciarse flexión, composición y derivación y, entre los afijos, prefijos y sufijos (flexivos y derivativos). Esta conclusión, válida para el inglés, debe matizarse para el español. Soto-Corominas (2021) señala que los niños adquieren la flexión de género en los nombres (especialmente animados) antes de los dos años, frente al dominio, por ejemplo, del sufijo -dor, que alcanzaría un uso productivo alrededor de los 3-4 años (Auza Benavides, Jackson-Maldonado y Maldonado, 1998). El uso productivo de las reglas de composición (por ejemplo, V-N en la formación de nombres de instrumento) no se daría hasta los 5 años (Álvarez et al., 1999).

Con relación al desarrollo de la conciencia morfológica, Roy y Labelle (2007) mostraron, para el francés, que los niños obtienen mejores resultados en las tareas experimentales de reconocimiento morfológico cuando los sufijos son frecuentes y productivos. Igualmente, obtienen mejores resultados con bases que no presentan alomorfos o particularidades ortográficas. Concluyen también que la noción de familia de palabras está plenamente adquirida en segundo año de Primaria.

También Salvo de Vargas e Isuani (2017) señalan, con relación al español, que, aun cuando las competencias receptiva y relacional 
están ya desarrolladas, las relaciones forma-significado opacas son difíciles de percibir por los niños. Así, la transparencia del derivado, la transparencia semántica del sufijo, la frecuencia del sufijo en la lengua oral, o que la base sea una palabra son factores que pueden afectar a la capacidad del niño para reflexionar sobre la morfología (véase también Olarte, 1985).

\section{5.- La enseñanza de la morfología en Primaria. Propuesta de secuenciación de contenidos}

De lo dicho hasta el momento pueden extraerse las siguientes conclusiones:

- La enseñanza explícita de la morfología en Educación Primaria resulta necesaria y oportuna en tanto que permite alcanzar los objetivos comunicativos de ampliación del vocabulario y mejora de la comprensión lectora y expresión escrita del alumnado.

- Para la consecución óptima de estos objetivos, resulta imprescindible basar la secuenciación de los contenidos en las conclusiones de estudios como los citados en las secciones anteriores. Asimismo, la enseñanza de la morfología deberá ser reflexiva y permitir al alumnado establecer generalizaciones e hipótesis significativas.

- El planteamiento de la actual ley educativa y su reflejo en los libros de texto debería ser revisado a partir de estas conclusiones.

En el primer apartado de este artículo, señalábamos varios problemas de la actual ley educativa, reflejados en los materiales de aula, para la consecución de los objetivos que en ella se formulan: la gran cantidad de contenidos que han de abordarse, la falta de progresión en su presentación, la ausencia de reflexión en las actividades de aprendizaje y su desconexión de la comunicación.

Un modo de paliar o evitar algunos de estos problemas es tener en cuenta los siguientes los hallazgos de la lingüística experimental: 
a) En español, la adquisición del componente morfológico se desarrolla secuencialmente, primero con (algunos aspectos de) la flexión, después con la derivación y por último con la composición.

b) La progresión en la enseñanza de la morfología ha de adecuarse a las capacidades reflexivas del niño. La conciencia morfológica comprende un conjunto de conocimientos a los que se accede de manera secuencial (Tyler y Nagy, 1989): conocimiento receptivo, conocimiento relacional, conocimiento sintáctico y conocimiento distribucional. Es razonable proponer que los contenidos morfológicos se introduzcan en Primaria de manera paralela al modo en que se desarrolla en el niño la capacidad de acceder a estos conocimientos.

c) Por último, debe considerarse que los resultados en tareas de reflexión son mejores cuando se utilizan bases y sufijos frecuentes que no presentan alomorfos (Roy y Labelle, 2007), y palabras derivadas cuyo significado es transparente (Salvo de Vargas e Isuani, 2017). Como nos señala un revisor anónimo, esto implica también adoptar una perspectiva sincrónica en la enseñanza de la morfología.

Teniendo en cuenta todo esto, presentamos en lo que sigue unos principios esquemáticos de secuenciación de contenidos que, a nuestro juicio, deberían ser un punto de partida para el tratamiento de los contenidos de morfología en Primaria.

Si Tyler y Nagy (1989) están en lo cierto, los niños entre 6 y 8 años son capaces de identificar unidades con significado menores de la palabra, pero no de identificar relaciones morfológicas entre dos o más palabras. Los primeros cursos de Primaria $\left(1^{\circ}-3^{\circ}\right)$ habrían de dedicarse, pues, a trabajar el concepto de palabra y la estructura interna de esta. Para ello podrá reflexionarse sobre palabras que tengan sufijos flexivos identificables (nominales o verbales, haciendo hincapié en las formas regulares), o sufijos derivativos, procesos ya adquiridos a esa edad. 
Con 9 años los niños poseen el conocimiento relacional necesario para identificar familias de palabras, conocimiento que debería introducirse a partir de tercer/cuarto curso.

Quedarían, de este modo, los dos últimos cursos de Primaria para trabajar el conocimiento sintáctico, que les permitirá identificar distintos procesos de formación de palabras. Dentro de este conocimiento, debe abordarse primero la derivación y, después, la composición.

Resulta necesario, además, hacer ciertas precisiones sobre la selección de ejemplos para la presentación de los contenidos y para las actividades:

- Durante toda la etapa de Primaria, pero especialmente para el desarrollo del conocimiento relacional, resulta imprescindible que el alumno trabaje en primer lugar con palabras que le resulten familiares y cuyo significado sea transparente a partir de su estructura morfológica: a modo de ejemplo, madrina no será percibida como una palabra derivada sino simple, igual que pasillo o mesilla, por lo que este tipo de formaciones deben evitarse.

- El conocimiento de familias de palabras resulta fundamental para el desarrollo del vocabulario; ha de escogerse, no obstante, de manera muy cuidadosa el conjunto de términos con el que se trabaja, de modo que el punto de partida lo formen palabras conocidas para posteriormente ir ampliando el vocabulario. El trabajo con familias de palabras, además, resultará fundamental en cursos posteriores para abordar los procesos de formación de palabras.

- La aproximación a los contenidos relacionados con la flexión debe hacerse teniendo en cuenta en todo momento que el estudiante es hablante nativo de la lengua que está estudiando; así pues, los morfemas flexivos no deben presentarse como listas cerradas que han de aprenderse, sino que ha de potenciarse la reflexión sobre estas marcas que ya conocen (el niño de quinto y sexto de Primaria ya "sabe" que cantaba es un tiempo de pasado 
frente a cantará, que es de futuro, y que la diferencia temporal la marcan los sufijos flexivos).

- Tanto para la composición como para la derivación, las palabras que se van a presentar han de escogerse atendiendo a distintos factores, ya mencionados anteriormente:

a) conviene que la nueva palabra sea semánticamente transparente, por lo que resulta más sencillo introducir la composición con palabras como lavavajillas que hacerlo con otras como nomeolvides;

b) la frecuencia de uso en el habla infantil de los ejemplos que se trabajen ha de ser también considerada: términos como subdelegado o subsuelo parecen más adecuados para explicar el prefijo sub- que otros atestiguados en libros de Primaria como subestimar, que no forma parte del vocabulario de niños de esta edad.

c) Es necesario tener también en cuenta otros procesos gramaticales que puedan interferir en la reflexión, por ejemplo, el hecho de que submarino, formado a partir de un prefijo y una base adjetiva, ha sufrido un proceso de sustantivación léxica. En este sentido, se deben tener en cuenta los factores que facilitan la percepción y el uso de las partes de las palabras, señalados por Serrano Dolader (2019:18): 


\section{Tabla 2}

Factores que facilitan la percepción y el uso de las partes de las palabras

\begin{tabular}{|c|c|c|}
\hline USO & $\begin{array}{l}\text { 1. Frecuencia } \\
\text { 2. Productividad } \\
\text { 3. Regularidad de } \\
\text { función }\end{array}$ & $\begin{array}{l}\text { - el afijo aparece en muchas palabras } \\
\text { - el afijo aparece en palabras } \\
\text { frecuentes } \\
\text { - el afijo sigue usándose para formar } \\
\text { nuevas palabras } \\
\text { - la palabra afijada pertenece a la } \\
\text { misma clase que la base } \\
\text { - el afijo se une a una base de una } \\
\text { clase determinada y produce una } \\
\text { palabra de una clase formal específica }\end{array}$ \\
\hline SIGNIFICADO & $\begin{array}{l}\text { 4. Transparencia } \\
\text { semántica } \\
\text { 5. Predictibilidad }\end{array}$ & $\begin{array}{l}\text { - los significados de base y afijo } \\
\text { están relacionados con el significado } \\
\text { de la palabra compleja } \\
\text { - el afijo tiene un único significado o } \\
\text { uno muy común } \\
\text { - el afijo tiene significado tanto } \\
\text { semántico como gramatical }\end{array}$ \\
\hline FORMA & $\begin{array}{l}\text { 6. Forma libre } \\
\text { 7. Regularidad oral } \\
\text { de la base } \\
\text { 8. Regularidad oral } \\
\text { del afijo } \\
\text { 9. Regularidad } \\
\text { escrita de la base } \\
\text { 10. Regularidad } \\
\text { escrita del afijo }\end{array}$ & $\begin{array}{l}\text { - la base es una palabra } \\
\text { - tal combinación de fonemas o de } \\
\text { letras solo forma un afijo } \\
\text { - la forma oral de la base no cambia } \\
\text { con la adición del afijo } \\
\text { - la forma oral del afijo no cambia al } \\
\text { adjuntarse } \\
\text { - la forma escrita de la base no } \\
\text { cambia al añadirse el afijo } \\
\text { - la forma escrita del afijo no cambia } \\
\text { al adjuntarse }\end{array}$ \\
\hline
\end{tabular}

Fuente: Serrano Dolader (2019, p.18)

Mención aparte merece la propuesta de actividades que ha de hacerse para desarrollar los contenidos. Escapa de los objetivos de este artículo presentar el tipo de ejercicios que se han de proponer en Primaria, pero, en la línea de lo señalado, habrán de ser tareas reflexivas y contextualizadas desde el punto de vista del uso real de la lengua. Ejercicios cuyo objetivo es unir un prefijo con una base dentro de listas predeterminadas para formar una nueva palabra tienen escaso valor en relación con las conclusiones extraídas en los apartados anteriores; 
deberían igualmente evitarse las tareas en que se presentan palabras descontextualizadas.

\section{Conclusiones}

En este trabajo se ha pretendido mostrar que la enseñanza explícita de la morfología, entendida como el desarrollo de la conciencia morfológica, puede y debe hacerse en la Educación Primaria. La idea que se ha querido transmitir, en la que se ha basado el esquema de propuesta esbozado, es que, para plantear la reflexión sobre la morfología en el aula de Primaria, deben tenerse en cuenta al menos los siguientes principios:

1. La organización de los contenidos morfológicos debe hacerse conforme a las conclusiones que nos ofrecen los estudios sobre adquisición y procesamiento del vocabulario. Específicamente, debe atenderse al hecho de que la conciencia morfológica del niño incluye poder acceder a distintos tipos de conocimientos, pero ese acceso no se desarrolla de forma simultánea ni uniforme: así, el trabajo del conocimiento receptivo (actividades de búsqueda y comparación) y del conocimiento relacional (familias de palabras) será central en la etapa de Primaria. El acceso al conocimiento sintáctico (y distribucional) solo podrá realizarse en los últimos cursos de esta etapa.

2. Los ejemplos que se utilicen han de seleccionarse $y$ graduarse progresivamente conforme a la frecuencia de uso de los afijos, rendimiento en el sistema, transparencia, composicionalidad de la forma completa y regularidad formal de bases y afijos.

3. La enseñanza de la morfología en Primaria debe tener como objetivo principal mejorar el vocabulario.

4. De la mano de lo anterior, la reflexión morfológica debe servir para mejorar la lectura y la escritura. Por ello, las tareas de reflexión morfológica no pueden presentarse aisladas, descontextualizadas, desligadas del trabajo de las 
destrezas (del trabajo de la ortografía, de la escritura, de la comprensión de textos...).

Además, como señala también Martín Vegas (2018) en su propuesta para trabajar la morfología en bachillerato:

5. La reflexión sobre la morfología debe basarse en conceptos morfológicos claros y bien establecidos en el campo (definición de palabra, morfema, raíz...), esto puede conllevar una reducción de los contenidos trabajados, aspecto que no se ha desarrollado en este artículo.

Habiendo sentado estos principios, y desarrollado un esqueleto de propuesta de secuenciación de contenidos, será necesario posteriormente detenerse en el diseño en sí de las actividades de reflexión (en la línea de las ideas desarrolladas por $\mathrm{GrOC}^{8}$ ) y no ser meros ejercicios de aplicación de definiciones e identificación mecánica de unidades linguísticas. Además, idealmente, las actividades de reflexión deberán estar insertas en esta etapa educativa en tareas más amplias de corte comunicativo centradas en el alumno.

Es, pues, patente que integrar la reflexión linguiística en el aula de Primaria no puede ser tarea exclusiva del maestro, sino que ha de abordarse desde equipos interdisciplinares que ofrezcan materiales adecuados. Estos equipos deberán incluir a lingüistas (morfólogos), a expertos en didáctica, pero también, crucialmente para la etapa de Primaria, a expertos en adquisición y en procesamiento de la lengua en la edad infantil. La reflexión sobre la lengua que exige la ley en esta etapa educativa no puede hacerse cabalmente sin los hallazgos de estas dos disciplinas. Si, además, como señala Querol Bataller (2015), la enseñanza de la morfología puede potenciarse utilizando una perspectiva interlingüística, queda aún un largo camino por recorrer dentro de esta línea de trabajo.

${ }^{8}$ Gramática orientada a las competencias: https://sites.google.com/view/groccast/ inicio 


\section{Referencias bibliográficas}

Álvarez, A., Casares, M. F., Olivares, M. A., y Zinkgräf, M. (1999). La adquisición de compuestos en el español: ¿un proceso tardío? Revista de Logopedia, Foniatría y Audiología, 19(4), 190-202.

Auza Benavides, A., Jackson-Maldonado, D., y Maldonado, R. (1998). Estrategias de productividad morfológica en el niño de tres a tres años y medio: el caso de los nombres de ocupaciones, Función, 18, $35-53$.

Ávila, R. (1991). Densidad léxica y adquisición del vocabulario: niños y adultos. En C. Hernández et al. (Eds.), El español de América. Actas del III congreso internacional del español en América, vol. 2, 621-630. Junta de Castilla y León, España. Recuperado de https://dialnet.unirioja.es/servlet/libro?codigo $=2688$

Berko, J. (1958). The child's learning of English morphology. Word, 14, 150-177.

Bloom, P. (2000). How children learn the meanings of words. Cambridge, MA: MIT Press.

Bloom, P. (2001). Précis of How children learn the meanings of words. Behavioral and Brain Scienceces, 24(6), 1095-1104. https://doi.org/10.1017/s0140525x01000139

Bosque, I. y Gallego, Á. (2016). La aplicación de la gramática en el aula. Recursos didácticos clásicos y modernos para la enseñanza de la gramática. Revista de Lingüística Teórica y Aplicada, 54(2), 6383.

Bosque, I. y Gallego, A. (2018). La gramática en la Enseñanza Media. Competencias oficiales y competencias necesarias. ReGroC, Revista de Gramática orientada a las competencias, 1, 141-202.

Butterworth, G. (1983) Structure of the mind in human infancy. Higher Education Quarterly, 37(3), doi: https://doi.org/10.1111/j.1468-2273.1983.tb01364.x

Caramazza, A., Laudanna, A., y Burani, C. (1988). Lexical access and inflectional morphology. Cognition, 28, 297-332. doi:10.1016/0010-0 277(88)90017-0

Carlisle, J.F. (1988). Knowledge of derivational morphology and spelling ability in fourth, sixth and eight graders. Applied 
Psycholinguistics,

9 , 247-266.

doi:

https://doi.org/10.1017/S0142716400007839

Carlisle, J.F. (1995). Morphological Awareness and Early Reading Achievement. En L. Feldman (Ed.), Morphological Aspects of Language Processing, 189-209. Hillsdale: Erlbaum.

Carlisle, J.F. y Stone, C.A. (2003). The effects of morphological structure on children's reading of derived words in English. En E.M.H. Assink y D. Sandra (Eds.), Reading Complex Words. Boston, MA: Springer. doi: https://doi.org/10.1007/978-1-47573720-2_2en

Clark, E. V. (2015). First Words. En J. R. Taylor (Ed.), The Oxford Handbook of the Word (cap. 30). Oxford: OUP. doi: 10.1093/oxfordhb/9780199641604.013.022

Currículo básico de Primaria (Real Decreto 126/2014, de 28 de febrero; BOE, número 52, 1 de marzo de 2014)

Forster, K.I. (1976). Accessing the mental lexicon. En R.J. Wales y E. Walker (Eds.), New Approaches to Language Mechanisms (pp. 257-287). Amsterdam: North-Holland.

Fromkin, V. (1985) (ed.). Phonetics Linguistics: essays in honor of Peter Ladefoged. Orlando: Academic Press.

Gómez Vera, G., Sotomayor, C., Bedwell, P., Domínguez, A.M., y Jéldrez, E. (2014). Análisis de la calidad del léxico y su relación con la calidad de la escritura en niños chilenos de cuarto año de educación Primaria. Documento de Trabajo nº 16 . Centro de Investigación Avanzada en Educación. Universidad de Chile.

Hernández Muñoz, N. (2015). La evaluación de la competencia léxica adulta: una aproximación a través de la disponibilidad léxica y la especialización académica en preuniversitarios. Revista de Filología de la Universidad de La Laguna, 33, 79-99.

Jaichenco, V. y Wilson, M. (2013). El rol de la morfología en el proceso de aprendizaje de la lectura en español. Interdisciplinaria, 30(1), 85-99.

Kernan, K.T. y Blount, B.G. (1966). The acquisition of Spanish grammar by Mexican children. Anthropological Linguistics, 8, 1-14. 
Martín Vegas, R.A. (2018). Modelos de aprendizaje léxico basados en la morfología derivativa. RILCE, 34(1), 262-85. doi: I0.15581/008. 34.1.262-85

McCutchen, D. (2011). From novice to expert: Implications of language skills and writing-relevant knowledge for memory during the development of writing skill. Journal of Writing Research, 3(1), 51-68.

McNamara, D., Scott, C., y McCarthy P. (2010). Linguistic Features of Writing Quality. Written Communication, 27(1), 57-86. doi: https://doi.org/10.1177\%2F0741088309351547

Morin, R. (2003). Derivational Morphological Analysis as a Strategy for Vocabulary Acquisition in Spanish. The Modern Language Journal, 87(2), 200-221.

Morton, J. (1979). Facilitation in word recognition: Experiments causing change in the logogen model. En P.A. Kolers, M.E. Wrolstad y H. Bouma (Eds.), Processing of visible language, 259268, Boston, M.A: Springer.

Nagy, W., Diakidoy, I-A., y Aderson, R. (1993). The acquisition of morphology: Learning the contribution of suffixes to the meanings of derivatives. Journal of reading Behavior, 25(2), 155-170.

Olarte, G. (1985). Acquisiton of Spanish Morphemes by Monolingual, Monocultural Spanish Speaking Children. Tesis Doctoral, University of Florida.

Querol Bataller, M. (2015). Propuestas para llevar la gramática a los alumnos. Tejuelo. Didáctica de la Lengua y la Literatura. Educación, 15, 141-167.

Raiter, A. y V. Jaichenco. (2002). Psicolingüística. Buenos Aires, Argentina: Editorial Docencia.

Rodrigo, M., Jiménez, J., García, E., Díaz, A., Ortiz, R., Guzmán, R., Hernández, I., Estévez, A., y Hernández, S. (2004). Valoración del procesamiento ortográfico en niños españoles con dislexia: El papel de las unidades léxicas y subléxicas. Electronic Journal of Research in Educational Psychology, 2(2), 105-126.

Rodríguez Montes, T. (2020). El desarrollo de la competencia léxico-semántica a través de la morfología léxica. Revista de Filología, 40, 275-288. doi: https://doi.org/10.25145/j.refiull.2020.40.13

Roy, C. y Labelle, M. (2007). Connaissance de la morphologie dérivationnelle chez les francophones et non-francophones de 6 à 8 
ans. Canadian Journal of Applied Linguistics, 10(3), 263-291. Recuperado de https://journals.lib.unb.ca/index.php/CJAL/article/view/19746

Salvo de Vargas, M. E. e Isuani, M. E. (2017). El desarrollo de la competencia morfoléxica en la educación Primaria. Evaluación de procesos derivativos de sufijación en español. Editorial Academia Española.

Sánchez Gutiérrez, C. (2012). ¿Cómo se da la descomposición morfológica en el procesamiento léxico? Una cuestión de etapas. ELUA Estudios de Lingüistica Universidad de Alicante, 26(26), 289-318. doi: 10.14198/ELUA2012.26.10

Saragossà, A. (2015). La morfología i els seus conceptes en l'ensenyament secundari: tractaments habituals. Tejuelo. Didáctica de la Lengua y la Literatura. Educación, 15, 168-190.

Serrano Dolader, D. (2019). Formación de palabras y enseñanza del español LE/L2. Londres y Nueva York: Routledge.

Soto Corominas, A. (2021). Morphology and L1 Acquisition. En A. Fábregas, V. Acedo-Matellán, G. Armstrong, M. C. Cuervo y I. Pujol-Payet (Eds.), The Routledge Handbook of Spanish Morphology (capítulo 36). London: Routledge.

Taft, M. y Forster, K.I. (1975). Lexical storage and retrieval of prefixed words. Journal of Verbal Learning and Verbal Behavior, 14, 638-647.

Tyler, A. y Nagy, W. (1989). The acquisition of English derivational morphology. Journal of Memory and Language, 28(6), 649-667. doi: https://doi.org/10.1016/0749-596X(89)90002-8 
Tejuelo, nº 35.1 (2022), págs. 71-106. El tratamiento de la morfología en Primaria...

106 | Pág in a 